\title{
Finite tidal effects in GW170817: Observational evidence or model assumptions?
}

\author{
W. Kastaun ${ }^{1,2}$ and F. Ohme $e^{1,2}$ \\ ${ }^{1}$ Max Planck Institute for Gravitational Physics (Albert Einstein Institute), Callinstr. 38, D-30167 Hannover, Germany \\ ${ }^{2}$ Leibniz, Universität Hannover, D-30167 Hannover, Germany
}

\begin{abstract}
After the detection of gravitational waves caused by the coalescence of compact objects in the mass range of neutron stars, GW170817, several studies have searched for an imprint of tidal effects in the signal, employing different model assumptions. One important distinction is whether or not to assume that both objects are neutron stars and obey the same equation of state. Some studies assumed independent properties. Others assume a universal equation of state, and in addition that the tidal deformability follows certain phenomenological relations. An important question is whether the gravitational-wave data alone constitute observational evidence for finite tidal effects. All studies provide Bayesian credible intervals, often without sufficiently discussing the impact of prior assumptions, especially in the case of lower limits on the neutron-star tidal deformability or radius. In this article, we scrutinize the implicit and explicit prior assumptions made in those studies. Our findings strongly indicate that existing lower credible bounds are mainly a consequence of prior assumptions combined with information gained about the system's masses. Importantly, those lower bounds are typically not informed by the observation of tidal effects in the gravitational-wave signal. Thus, regarding them as observational evidence might be misleading without a more detailed discussion. Further, we point out technical problems and conceptual inconsistencies in existing studies. We also assess the limitations due to systematic waveform model uncertainties in a novel way, demonstrating that differences between existing models are not guaranteed to be small enough for an unbiased estimation of lower bounds on the tidal deformability. Finally, we propose strategies for gravitational-wave data analysis designed to avoid some of the problems we uncovered.
\end{abstract}

PACS numbers: 04.25.dk, 04.30.Db, 04.40.Dg, 97.60.Jd,

\section{INTRODUCTION}

Among known astronomical objects, the highest matter densities occur within neutron stars (NSs). The behavior of cold matter at supranuclear densities cannot be studied in laboratories on Earth. Therefore, fundamental properties such as the equation of state (EOS) that relates pressure, temperature, and density, are essentially unconstrained by experiments. Theoretical models calibrated by properties of atomic nuclei can be used to extrapolate the EOS of neutron-star matter, but face considerable theoretical uncertainties. Astrophysical observations of NSs can be used instead to infer EOS constraints, based on the EOS impact on NS structure and dynamics.

Merging NS binaries in particular are an excellent environment to probe the EOS, since the gravitational wave $(\mathrm{GW})$ signal emitted before, during, and after merger carries signatures of the EOS, as do electromagnetic (EM) counterparts given by kilonova and short gamma ray burst. The first multimessenger observation [1-3] of a binary neutron star (BNS) merger event, GW170817, indeed opened a wealth of opportunities.

The most direct observable signature of the EOS is contained in the GW signal emitted during the last seconds before the merger. While a neutron star and another compact object spiral around each other, the neutron star is deformed in the tidal field of the companion. This deformation modifies the dissipation of energy and angular momentum by GW and thereby affects the decay of the orbit. Measuring the inspiral frequency evolution therefore carries imprints of the EOS [4], in addition to the constituents' masses and angular momenta.

For binary neutron stars, the frequencies during this phase fall within the sensitive range of current ground-based interferometers, such as the Advanced Laser Interferometer
Gravitational-Wave Observatory (LIGO) [5] or the Virgo detector [6]. In contrast, merger and postmerger GW emission is dominated by frequencies $>1 \mathrm{kHz}$, for which the current detection range is considerably smaller, making observations of this phase unlikely (compare [7--9]).

For the first observation of GWs from a binary NS merger, GW170817 [1], several studies have already presented constraints on the tidal parameters that enter the GW signal [7. 10-13]. All of those provide robust upper limits on the tidal effects. Although more difficult to measure, lower bounds are provided as well.

In this paper, we revisit the methods used in [7, 10, 11] to derive such constraints. We scrutinize the implicit and explicit assumptions involved, in order to decide if the published lower limits can be interpreted as direct observational evidence of finite tidal effects, or rather constitute inferences based on model assumptions and observational constraints on the masses. The latter would be the case when the same (or very similar) limits are obtained regardless of whether the real source was a neutron star or black hole $(\mathrm{BH})$ binary.

We emphasize that we do not argue against combining observational data and prior knowledge, e.g., from nuclear physics, in order to provide tighter constraints on the EOS. Before interpreting such constraints as observational evidence, however, the contribution of model assumptions needs to be accounted for. In any case, if the model assumptions include ambiguous ad-hoc choices or inconsistencies, then resulting constraints are only meaningful to the extent that they are dominated by the observational data and not the prior knowledge. We review prior choices with regard to the above.

Before explaining the main challenges, we briefly review the Bayesian framework that is typically used to infer the properties of binary GW sources (see [14]). The binary's 
vector of parameters, $\theta$ (composed of the binary NS properties such as masses, distance, inclination, etc., and also EOSrelated parameters) is determined in the form of its posterior probability density,

$$
p(\theta \mid d, I)=\frac{P(d \mid \theta, I)}{P(d \mid I)} p(\theta \mid I),
$$

given the observed data $d$ and any additional model assumptions $I$. Bayes' Law, Eq. (1), relates the probability density distribution on the left-hand side to the product of the likelihood of the observed the data given the source parameters, $P(d \mid \theta, I)$, and the prior probability density of encountering a system with those parameters, $p(\theta \mid I)$. The likelihood is calculated by comparing gravitational waveform models with the data, assuming stationary Gaussian noise. The prior represents any knowledge or belief that existed before the data were obtained.

The main conceptual challenges all such Bayesian EOS analyses of binary NS GW data face are the following:

1. Parameterization of the unknown EOS. GW models depend on two parameters related to the tidal deformability of each object in the binary. If a common but unknown EOS is assumed, relating those two numbers is nontrivial. In Secs. II C and III C, we discuss model assumptions [15] (often referred to as "universal relations") that have been used to correlate masses and tidal deformabilities of NS binaries, analyze their range of applicability as well as pathologies arising from their use, and suggest a simpler alternative.

2. Using the posterior to claim observational evidence for tidal effects. Even for a completely unconstraining measurement, Bayesian methods provide a posterior distribution, which then only reflects the chosen prior distribution. To assess the contribution of observational data, one has to compare posterior and prior. This becomes nontrivial in a multidimensional parameter space. If the marginalized posterior for tidal effects is constrained in comparison to the prior, it still does not signify a direct measurement of tidal effects. The reason is that the data might only constrain the NS masses. If the prior distribution for tidal effects depends on the masses, the posterior is then influenced even without any constraint of tidal effects. We therefore separate the indirect impact of observational constraints on quantities that are unrelated to tidal effects from the impact of those that are directly related. For this, we discuss idealized cases such as fixed mass ratios.

3. Ambiguity of the prior probability for the EOS-related quantities. The choice of prior is a well-known issue in Bayesian analysis. There are no other observations that could be used as prior knowledge on tidal deformability, and the requirement to specify a prior distribution can only be satisfied by ambiguous ad-hoc choices. We examine the shape of priors used for published EOS constraints in detail and discuss implications.

4. Credible bounds do not imply statements on likelihood of particular models. Depending on the shape of the posterior, a specific model with parameters outside the credible bounds can be more likely than another model within the bounds. Even the maximum likelihood parameter can be located outside a given credible interval. It is always possible to compute a two-sided credible interval, which, on its own, is therefore not useful as evidence against specific models. For this, Bayesian model selection is more suitable. A recent model selection study on GW170817 [16] did not find evidence for or against finite tidal effects. In this work, we do not compute Bayes factors, but provide arguments, based on features of the posterior and prior distributions, against attributing the lower credible bounds to observational evidence.

5. Theoretical uncertainty of waveform model. Computing the likelihood requires a theoretical model of the gravitational waveform for given parameters. The systematic error of the latter is currently not known, but estimated by comparing different theoretical models [17]. Further, estimating the resulting systematic error of credible bounds based on statistical analysis of a given event is complicated by the presence of the waveformindependent statistical errors. In Sec. IIIF we provide a new perspective on estimating the potential impact of systematic waveform errors. The order of magnitude we find for the systematic errors indicates that lower bounds on tidal parameters should be interpreted with great caution.

The issues discussed in this work are also relevant when planning the data analysis strategy for future events. This involves a choice of how many model assumptions to incorporate already during the GW data analysis, and which quantities to provide for comparison to nuclear physics models. In Sec. III G, we propose a strategy that keeps the division line between data analysis and neutron-star modeling as close to the measurable quantities as possible, which simplifies the interpretation of results and allows one to study the consequences of different model assumptions without repeating computationally expensive parameter estimation studies. Employing a reasonable strategy will be important to facilitate the exciting synergy of GW astronomy and nuclear physics as more observations become available.

\section{BINARY NEUTRON-STAR PARAMETERS}

\section{A. Masses}

The evolution of the GW signal during the inspiral depends most strongly on a combination of the individual gravitational masses $M_{1}$ and $M_{2}$ called chirp mass,

$$
M_{c}=\frac{\left(M_{1} M_{2}\right)^{3 / 5}}{\left(M_{1}+M_{2}\right)^{1 / 5}} .
$$

For a BNS merger, the chirp mass can be determined very accurately by current $\mathrm{GW}$ detectors. We therefore treat it as 
fixed in this article. We specifically use the value $M_{c}=1.186$ inferred for GW170817 [7]. The mass ratio $q=M_{2} / M_{1}$ is less well constrained. For the given chirp mass, we can express the individual NS masses as a function of the mass ratio,

$$
\begin{aligned}
& M_{1}(q)=M_{c}(1+q)^{1 / 5} q^{-3 / 5}, \\
& M_{2}(q)=M_{c}(1+q)^{1 / 5} q^{2 / 5} .
\end{aligned}
$$

We focus on the simple case of vanishing NS spins in this work, although they can have a significant influence on the GW signal. The particular challenges that we discuss are not reduced when allowing for spins, while ignoring spins considerably simplifies the discussion. We also make the common assumption of negligible orbital eccentricity.

\section{B. Effective tidal deformability}

In the context of GW signal, the difference between NS and $\mathrm{BH}$ cases is how the objects' quadrupole moments react to the tidal forces caused by their companion. This is expressed by the dimensionless tidal deformabilies $\Lambda_{1}, \Lambda_{2}$ of the two objects, which are 0 for BHs (see [18] however). Since we neglect the NS spins, finite size effects are given by the tidal deformability as a function of gravitational mass alone, $\Lambda(M)$, which depends on the EOS. The correction to the point-particle GW signal mainly depends on a combination (see [4]) called effective tidal deformability,

$$
\tilde{\Lambda}=\frac{16}{13} \frac{(1+12 q) \Lambda_{1}+(q+12) q^{4} \Lambda_{2}}{(1+q)^{5}} .
$$

In this work, we use the rescaled quantity

$$
\begin{aligned}
\bar{\Lambda} & =\tilde{\Lambda} w_{f}^{-1}(q), \\
w_{f}(q) & =\frac{16}{13} \frac{1+12 q+(q+12) q^{4}}{(1+q)^{5}} .
\end{aligned}
$$

The interpretation of $\bar{\Lambda}$ is convenient because for a BNS, it results from a linear interpolation of $\Lambda(M)$ between the masses $M_{1}$ and $M_{2}$, evaluated at a mass $\bar{M}$ defined by

$$
\begin{aligned}
\bar{M}(q) & =w_{1}(q) M_{1}(q)+w_{2}(q) M_{2}(q), \\
\bar{\Lambda}(q) & =w_{1}(q) \Lambda\left(M_{1}(q)\right)+w_{2}(q) \Lambda\left(M_{2}(q)\right) .
\end{aligned}
$$

For mass ratios close to unity, $\bar{\Lambda} \approx \Lambda(\bar{M})$. The weights depend only on the mass ratio, as

$$
\begin{aligned}
& w_{1}(q)=\frac{1+12 q}{1+12 q+(q+12) q^{4}} \\
& w_{2}(q)=\frac{(q+12) q^{4}}{1+12 q+(q+12) q^{4}} .
\end{aligned}
$$

where $w_{1}+w_{2}=1$. We follow the convention $q<1$, and therefore $w_{1} \geq 1 / 2$ and $w_{2} \leq 1 / 2$. Note $\bar{M}(q)$ is monotonically decreasing, which allows us to express $\bar{\Lambda}$ as a function of $\bar{M}$.
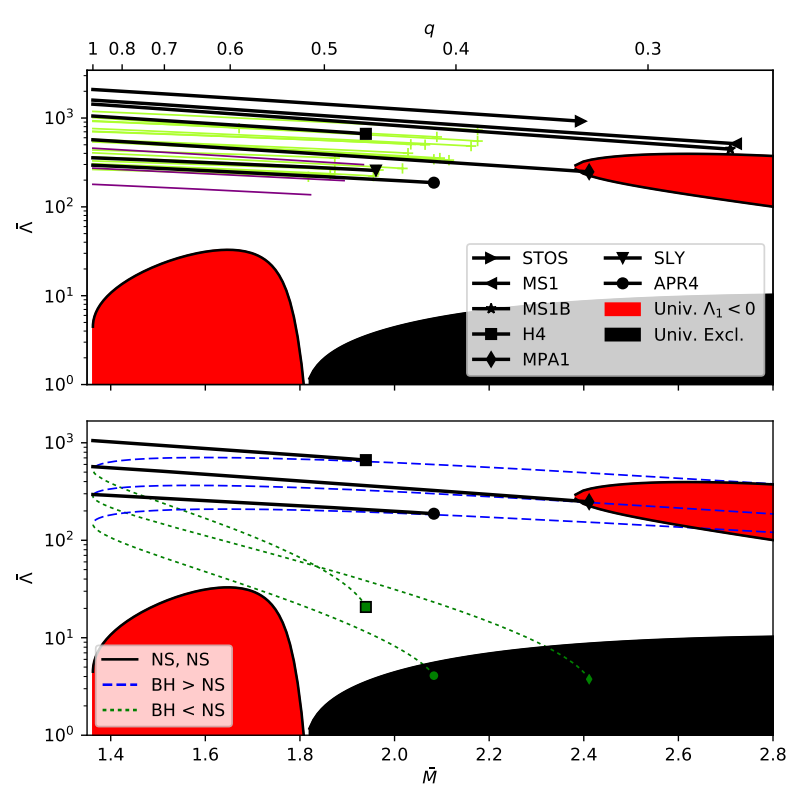

FIG. 1. Top: Interpolated tidal deformability $\bar{\Lambda}$ of a BNS system as a function of $\bar{M}$ (bottom axis) and $q$ (top axis). The curves correspond to various EOS, where only a representative set is labeled and the others are shown in green. Causality-violating EOS used in [10] are shown in purple. The sequences terminate when $M_{1}(q)$ reaches the maximum mass (marked by symbols). The shaded areas are regions unreachable when using the universal relations from [10], either implying negative tidal deformabilites (red) or by construction (black). Bottom: Comparison of $\bar{\Lambda}(\bar{M})$ between the BNS case and mixed NS-BH case, for three EOS. The solid curves refer to the BNS case, the dashed (dotted) curves to a mixed NS/BH binary where the heavier (lighter) object is the $\mathrm{BH}$.

For the BNS case with both NS obeying the same EOS, $\bar{\Lambda}$ is shown in the upper panel of Fig. 1 for a representative set of EOS. Note that the function is defined only up to a maximum $\bar{M}$ (minimum $q$ ) for which $M_{1}$ exceeds the maximum allowed mass for a nonrotating NS for the given EOS. For the case of a mixed binary, we can compute $\bar{\Lambda}$ by setting either $\Lambda_{1}$ or $\Lambda_{2}$ to 0 in Eq. 5). The resulting differences are shown in the lower panel for Fig. 1

Although only $\bar{\Lambda}$ can be confined by the GW signal, the difference of the tidal deformabilities of the two NSs is an important property of the EOS as well. In the following, we express it in terms of a dimensionless parameter

$$
\bar{S}=\frac{\ln \left(\Lambda_{1}\right)-\ln \left(\Lambda_{2}\right)}{\ln \left(M_{1}\right)-\ln \left(M_{2}\right)}=\frac{\ln \left(\Lambda_{2} / \Lambda_{1}\right)}{\ln (q)} .
$$

For moderate mass ratios, $\bar{S}$ has an intuitive interpretation in terms of the slope of $\Lambda(M)$,

$$
\bar{S} \approx \frac{\mathrm{d} \ln (\Lambda)}{\mathrm{d} \ln (M)}(\bar{M}) .
$$

In the context of waveform models, it is more common to use a quantity $\delta \tilde{\Lambda}$, which enters at higher post-Newtonian or- 
der than $\tilde{\Lambda}$ and has minor impact on the waveform [17]. For fixed $\bar{\Lambda}$ and $q$, the quantity $\delta \tilde{\Lambda}$ becomes a function of $\bar{S}$, which therefore has minor impact as well. Unless noted otherwise, we discuss the idealized case that the GW signal depends on $M_{c}, q, \bar{\Lambda}$ but not $\bar{S}$.

\section{Universal relations}

In order to make statements about the individual tidal deformabilites, the measurement of $\bar{\Lambda}$ is not sufficient; the slope $\bar{S}$ is required as well. Since it does not enter the GW signal, it cannot be measured. One way to infer properties of the individual NSs regardless of this fact is to use model-dependent assumptions about $\bar{S}$.

As proposed in [15], one can express the effective tidal deformability $\tilde{\Lambda}$ as an EOS-independent function of the average deformability and the mass ratio. The residual error (computed in [15]) of the expression in comparison to the results for a few nuclear physics EOS models is small enough to motivate the term universal relation.

In detail, the relations are written as $\Lambda_{a}\left(\Lambda_{s}, q\right)$, where $\Lambda_{s}=$ $\left(\Lambda_{1}+\Lambda_{2}\right) / 2$ and $\Lambda_{a}=\left(\Lambda_{2}-\Lambda_{1}\right) / 2$. The function $\Lambda_{a}$ is expanded in $\Lambda_{s}$ and $q$ as

$$
\begin{aligned}
\Lambda_{a} & =\Lambda_{s} F_{n}(q) \frac{1+\sum_{l=1}^{3} \sum_{k=1}^{2} b_{l k} q^{k} \Lambda_{s}^{-l / 5}}{1+\sum_{l=1}^{3} \sum_{k=1}^{2} c_{l k} q^{k} \Lambda_{s}^{-l / 5}} \\
F_{n}(q) & =\frac{1-q^{10 /(3-n)}}{1+q^{10 /(3-n)}} .
\end{aligned}
$$

Those relations are used in [10] for data analysis of GW170817. The coefficients $n, b_{i j}, c_{i j}$, given in [19], were obtained by fitting to a set of nuclear physics EOS. We noticed that the coefficients differ from those given in [15] (after taking into account that the definitions differ by inclusion of a scaling factor). However, the qualitative statements we make on universal relation usage are equally valid for both.

The set of EOS used for calibration in [10, 19] consists of SLY [20], LS220[21], Shen [22] (here:STOS), AP3 and AP4 [23], WFF1 and WFF2 [24], ENG [25], MPA1 [26], MS1 and MS1b [27]. We note that the AP3, AP4, WFF1, WFF2, and ENG EOS start to violate causality before reaching the central density of the maximum mass nonrotating NS solution. It is also worth pointing out that the set of EOS models used for calibration has maximum NS masses compatible with the observation of PSR J0348+0432 [28] (although for the WFF1 EOS, causality is violated at this mass). Since low tidal deformabilities typically occur near the maximum mass model and increase with decreasing mass, nuclear physics EOS candidates compatible with PSR J0348+0432 do not yield low deformabilities in the mass range measured for GW170817 (for the EOS set above, $\Lambda>61$ in the mass range inferred for the low spin prior in [7]; compare Fig. 1 in [16]). On the other hand, strong phase transitions are not considered and might alter the behavior of tidal deformability.

The universal relations $\Lambda_{a}\left(\Lambda_{s}, q\right)$ are shown in Fig. 2 in terms of our $\bar{\Lambda}, \bar{S}$ parametrization. The relations are evaluated for our fixed fiducial chirp mass, and various mass ratios in the range $0.4 \ldots 1$. For comparison, we plot the values $\bar{\Lambda}, \bar{S}$ for a representative set of EOS. As one can see, none of the EOS reach low values of $\bar{\Lambda}$ for the mass range of the detected event. Using the universal relations at lower values of $\bar{\Lambda}$ is self-contradictory, since the EOS used to derive the relations explicitly exclude such values. We note a subtle point: the models used in [15] to fit the universal relations do include lower values of $\bar{\Lambda}$. The reason is that the mass range of the models for the fit is not restricted. One should however keep in mind that the resulting fit around any given $\bar{\Lambda}$ is only valid within a range for $\bar{M}$ allowed by the EOS models. In other words, there might be EOS leading to low $\bar{\Lambda}$ for the detected mass range, but these EOS must then be very different from those used in the fit. In turn, the error of $\bar{S}$ from the universal relations is completely unknown in that case.

Besides the conceptual problem above, the relations used in [10] exhibit some technical problems. For low values of $\Lambda_{s}$, the expression Eq. (14) has a pole and ceases to be meaningful. The corresponding slope $\bar{S}$ diverges. The divergence leads to gaps in the possible values of $\bar{\Lambda}$. However, the divergence only affects very small values. More important is the fact that $\Lambda_{1}$ computed from the universal relation crosses 0 (we recall that negative tidal deformabilities are unphysical [29]). At this point $\bar{S}$ diverges since it is defined in a way that does not allow negative deformabilities. This happens below some critical $\bar{\Lambda}$ that depends on the mass ratio. Even before $\Lambda_{1}$ crosses 0 , the slope $\bar{S}$ greatly exceeds the range given by the EOS models, as shown in Fig. 3 Assuming the universal relations to hold universally thus excludes small effective tidal deformabilities. The excluded region is shown in Fig. 1 as a function of $\bar{M}$. Although not relevant for the case of GW170817, we note that for mass ratios $q \lesssim 0.5$, low values of $\bar{\Lambda}$ are excluded because the universal relation develops a minimum. For even more extreme mass ratios, a region at large $\bar{\Lambda}$ becomes invalid because $\Lambda_{1}<0$.

One could argue that the excluded regions at small $\Lambda$ follow from the universal relations, which hold to good accuracy for known nuclear physics EOS. However, the EOS used to derive the relations already exclude even larger $\Lambda$, as discussed earlier. The exact extent of the region excluded by negative $\Lambda_{1}$ is just the result of extrapolating a fit and has no physical meaning.

A similar set of universal relations is used in [11]. The set of EOS used for calibration consists of many piecewise polytropic EOS, each of which leads to maximum NS masses above $2 M_{\odot}$. For the mass range of the detected event, the tidal deformability of NS following those EOS is approximately proportional to $q^{6}$. This leads to a parametrization $\Lambda_{1}=\Lambda_{0} q^{3}, \Lambda_{2}=\Lambda_{0} / q^{3}$, where $\Lambda_{0}$ is a free parameter. In our notation, the slope parameter is $\bar{S}=6$, independent of $\bar{\Lambda}$ and $q$. The given slope parameter is also shown in Fig. 2. The differences to NS models with nuclear physics EOS are comparable to those found for the universal relation used in [10]. In contrast to [10], the relation used in [11] does not exhibit any divergence or excluded regions at low values of $\Lambda$.

It has become customary to express constraints on the EOS from NS observations in a mass-radius diagram. However, the radius does not enter the gravitational waveform models 


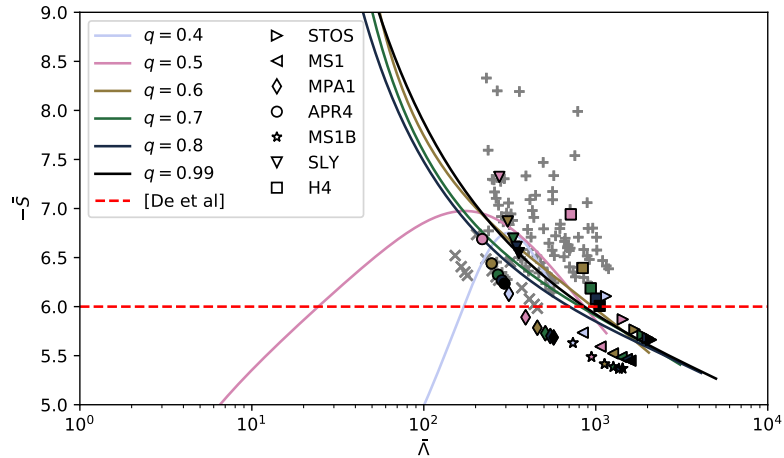

FIG. 2. Universal relations from [10 19], evaluated for fixed chirp mass. The solid curves show the parameters $-\bar{S}, \bar{\Lambda}$ resulting for fixed mass ratios, varying $\Lambda_{s}$ up to 5000 (the upper limit used in [19]). Note at very small $\Lambda_{s}$, there is an additional branch with $\bar{S}>0$ (not shown) due to the poles of the universal relations. The dashed line represents $-\bar{S}$ from [11], which does not depend on $q$ or $\bar{\Lambda}$. The labeled symbols mark the values computed for a representative set of nuclear physics EOS, colored according to mass ratio like the universal relation curves. Grey plus symbols show additional EOS, and grey crosses mark causality-violating EOS used in [10, 19].

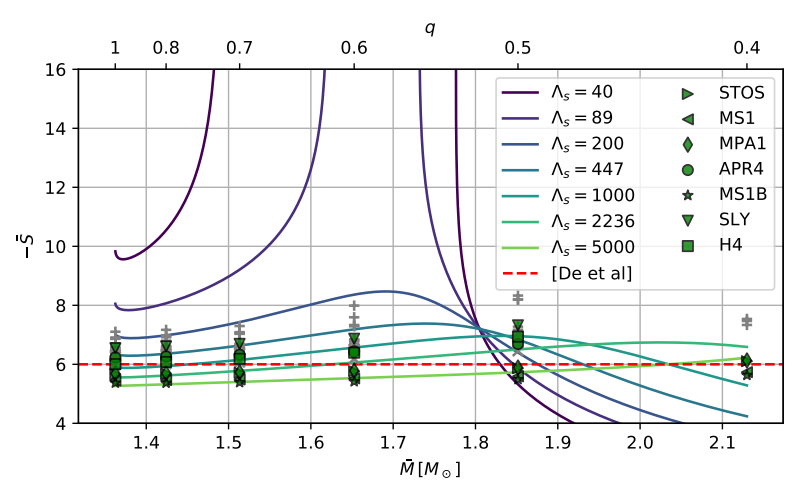

FIG. 3. Universal relations from [10 19], evaluated for fixed chirp mass. The curves show the slope parameter $\bar{S}$ versus $\bar{M}$ for fixed values of $\Lambda_{s}$ and varying mass ratio. The curves diverge where $\Lambda_{1}$ becomes negative. The symbols mark the values obtained for the same EOS as in Fig. 2 .

at all and therefore it cannot be measured. In [10] and [11], the NS tidal deformabilities were therefore converted to radii using another type of "universal" relation, which relates tidal deformability and compactness $C=M / R$ of the NS.

The universal $\Lambda-C$ relation used in [10] is given in [15, 19], while [11] introduces a different one. Both variants are shown in Fig. 4 For comparison, we plot values for our standard set of EOS at two masses spanning the range of the detected event. The universal relation $C_{u}(\Lambda)$ contains the same conceptual trap as the relation $\Lambda_{a}(\bar{\Lambda}, q)$ : for a given NS mass, it is only meaningful in the range covered by the set of EOS used for the fit. Outside this range, none of those mod-

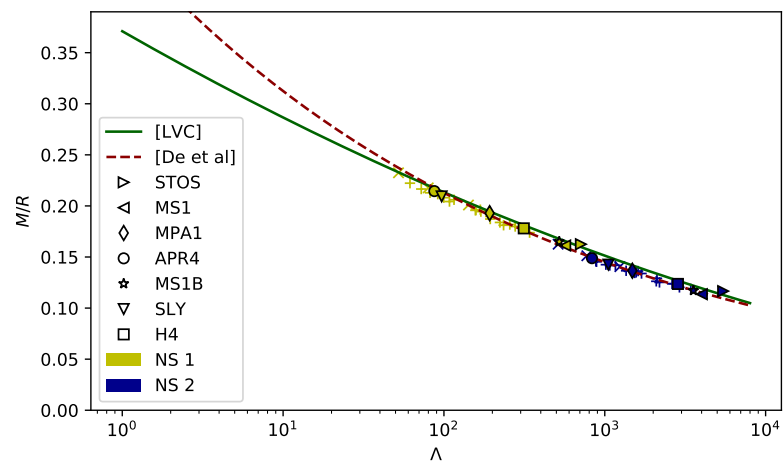

FIG. 4. Universal relation used in [10] for NS compactness as a function of tidal deformability (green curve). The dashed red curve shows a similar universal relation used in [11]. The labeled symbols and plus markers show values obtained from various EOS (the crosses correspond to causality-violating EOS) for the masses of the lighter (blue) and heavier (yellow) NS in GW170817 when assuming a mass ratio $q=0.7$.

els has the correct mass. For a NS with given mass and low tidal deformability, one cannot derive any expectation for the compactness from nuclear physics EOS if the very same set of EOS firmly excludes NS models with those $\Lambda, M$. We note that outside the range supported by NS models, the two relations differ significantly, since the relation used in [11] was only designed to fit the mass range of GW170817.

For each of the two masses shown in Fig. 4, the range of EOS spans only a limited range in $\Lambda$. For the example at mass $1.14 M_{\odot}$ (blue markers), the universal relation prediction for the compactness becomes increasingly unreliable below $\Lambda \lesssim 500$, the lower limit of the range allowed by the EOS set. This corresponds to a radius of $\approx 10 \mathrm{~km}$ when using the universal relation. NSs of same mass but lower tidal deformability would require an EOS very different from those used to construct the universal compactness relation. A possible cause for such qualitatively different behavior might involve phase transitions or quark matter, none of which are considered in the EOS sets used for calibration in [10, 11].

In conclusion, assuming that the EOS of an observed NS falls in the range of EOS used to calibrate the universal compactness relations already leads to lower limits of radius and tidal deformability based on the measured mass range alone. Inserting any measured tidal deformability below this limit in the universal relations to obtain a radius is contradictory, because the existence of such a NS invalidates the foundations of the relation.

A consistent use of universal relations in statistical analysis would require specifying a mass-dependent validity range defined by the set of EOS used to calibrate the relations, and to limit the prior support to valid regions in parameter space. Most likely, the resulting lower limits for GW170817 would then be close to the model-dependent ones already resulting from the validity region and the measured mass range. 


\section{APPLICATION TO DATA ANALYSIS}

\section{A. Ideal measurements}

In the following we study the constraints given by an exact measurement of the waveform. Although real measurements only yield statistical probabilities due to the noise of the detector, it is instructive to discuss this idealized case first, since real measurements can never be more constraining. We thus consider the case that the inspiral GW signal was measured with perfect accuracy and agrees exactly with a waveform model with parameters $q, \tilde{\Lambda}$. As always, we assume a fixed chirp mass, zero spins, and that $\bar{S}$ has negligible impact on the waveform.

The exact measurement of $q, \tilde{\Lambda}$ corresponds to a single point in the $(\bar{\Lambda}, \bar{M})$ parameter space shown in Fig. 1 A given EOS is compatible with the measurement if the corresponding function $\bar{\Lambda}(\bar{M})$ passes through the point. Note that for some of the nuclear physics EOS, the $\bar{\Lambda}(\bar{M})$ curves shown in the upper panel of Fig. 1 cross. With a single measurement of a neutron star binary at such an intersection, the two EOS would be completely indistinguishable. On the other hand, the curves for our set of EOS cross at shallow angles, such that the $\bar{\Lambda}(\bar{M})$ curves remain similar when moving away from the intersection point. How large the differences of two EOS away from an intersection point can become is a highly relevant open question.

The similarity between intersecting $\bar{\Lambda}(\bar{M})$ curves does not extend to maximum mass NS, however. EOS models with otherwise very similar curves can have very different maximum masses. Counterexamples visible in Fig. 1] show that the maximum NS mass cannot be determined from a single inspiralphase GW signal alone, although $M_{1}$ constitutes a lower limit (assuming slow rotation).

In general, the inverse problem of deducing the constraints on the actual EOS function $P(\rho)$ from $\bar{\Lambda}$ measured at one or several $\bar{M}$ is a very nontrivial problem, partly because the full parameter space of $P(\rho)$ has infinite dimension. However, it is not necessary to solve this problem for GW data analysis. Since the only information the GW signal carries is encoded in $\bar{\Lambda}, \bar{M}$, one could restrict signal analysis to this information. Testing model assumptions for compatibility or deriving information not in the GW signal by adding model assumptions can both be accomplished in a step separate from GW data analysis.

For an ideal measurement of $\bar{\Lambda}$ at some mass ratio $q$, the universal relations $\Lambda_{a}\left(\Lambda_{s}, q\right)$ yield exact values of the individual $\Lambda_{1}, \Lambda_{2}$, unless $\bar{\Lambda}$ is inside one of the excluded regions discussed in Sec. [IC. In our notation, the universal relation $\Lambda_{a}\left(\Lambda_{s}, q\right)$ can be written as $\bar{S}_{u}(\bar{\Lambda}, q)$, with a range $\bar{\Lambda}<\bar{\Lambda}_{e}(q)$ excluded by construction. The accuracy of $\Lambda_{a}\left(\Lambda_{s}, q\right)$ needs to be specified as part of the model assumptions. Since there is only one true EOS and one universal relation, the error is systematic. From the viewpoint of measurements, the model assumption is defined by the allowed range for $\Lambda_{a}$. A reasonable range is given by the largest fit residuals of the EOS used for calibrating the relations, although it depends on the selection of EOS models, which is part of the assumption. A compari- son of the slope parameter $\bar{S}$ between universal relations and various EOS is shown in Fig. 22 As one can see, the EOS we consider fulfill $4<-\bar{S}<9$. This range could serve as a simpler replacement for the use of universal relations $\Lambda_{a}\left(\Lambda_{s}, q\right)$ together with comparable accuracy assumptions.

Figure 2 also leads us to the following observations. First, the more complicated universal relations used in [10] do not reduce the residuals considerably compared to the more robust $\bar{S}=6$ prescription used in [11]. Further, the spread for the EOS we considered is somewhat larger than for the smaller set used to calibrate the universal relations in [10]. Finally, $\bar{S}_{u}(\bar{\Lambda}, q)$ diverges for $\bar{\Lambda}$ outside the range allowed by the EOS models considered here, and the error is essentially unknown. Using the same error as for $\bar{\Lambda}$ values compatible with the EOS models is not a well-motivated assumption, given that the divergence is caused by extrapolation of a particular form of the fitting function.

\section{B. Bayesian analysis}

The study of gravitational-wave data relies on Bayesian statistical methods. The main ingredients are (i) a theoretical model of the signal expected for given source and extrinsic parameters; (ii) a characterization of the detector noise that allows one to estimate how likely a given stretch of data contains a given signal; and (iii) the prior probability distribution for the source parameters, which encodes the state of knowledge before any measurement.

The choice of a prior is a very ambiguous ingredient, although there are some well-motivated constraints to start with. For example, gravitational masses of NS are positive in general relativity. Similarly, negative tidal deformabilities are usually excluded (see [29]). Another important decision is whether to assume that the EOS of NS is universal, i.e. that both NS follow the same $\Lambda(M)$. Further, the use of universal relations is equivalent to a particular choice of prior.

There is however no observational or theoretical knowledge that could motivate a choice for the exact shape of the tidal deformability prior distribution. In this situation, it is common to express the lack of knowledge by assuming a uniform prior within the bounds. However, this depends on the choice of parametrization and is therefore still ambiguous. For a NS, both $\Lambda$ and $\log (\Lambda)$ are natural choices. A further complication is given by the choice of variables for the multidimensional parameter space of a binary system, in particular, in connection with universal relations. We do not try to argue which prior is "correct" but describe the various priors used in previous studies and their implications in detail.

The studies [7, 10, 11] provide results on the tidal deformabilities in terms of the posterior distributions, which combine the prior with the information added by the observational data. The main results are one- or two-sided credible intervals on the tidal deformability or derived quantities. In the following sections, we investigate how much of the posterior depends on the prior assumptions, and how much information is added by the observational data.

With decreasing deformability, its impact on the waveform 
diminishes. For a given signal-to-noise ratio, we expect that there is some scale $\tilde{\Lambda}_{t}$ below which the ability of the detector to distinguish tidal effects gradually vanishes. One should therefore expect that any lower limit depends more strongly on the prior the smaller its value, and that upper limits depend less on the prior than lower limits.

With this motivation, we scrutinize the lower credible bounds that are already given by the priors. To this end, we introduce a thought experiment we dubbed the uninformative detector test. Consider a detector that measures with perfect accuracy the NS masses and whether or not the effective tidal deformability $\tilde{\Lambda}$ is greater than some threshold $\tilde{\Lambda}_{t}$ or not, but does not provide the value of $\tilde{\Lambda}$. This is an idealized model of a real detector, for which the ability to distinguish increases gradually instead of abruptly. The posterior obtained by a hypothetical measurement with the uninformative detector is given by the prior, after cutting it off above $\tilde{\Lambda}_{t}$ and normalizing it to unity (assuming the true value is below $\tilde{\Lambda}_{t}$ ).

Of course one can compute perfectly valid two-sided credible intervals $\left(\tilde{\Lambda}_{t}^{0}, \tilde{\Lambda}_{t}^{1}\right)$ for this posterior. However, the lower limit $\tilde{\Lambda}_{t}^{0}$ is rather useless because it is entirely given by the prior and the threshold $\tilde{\Lambda}_{t}$. By construction, the detector cannot distinguish any deformabilities below $\tilde{\Lambda}_{t}$, including 0 . A measurement with such a detector could never be considered as observational proof of finite tidal effects (unless the true value is above $\tilde{\Lambda}_{t}$ ).

The lower limit $\tilde{\Lambda}_{t}^{0}$ can serve as a reasonable estimate for the scale below which lower limits are likely not informed by the data, but the prior. As further motivation consider the likelihood $P(d \mid \theta, I)$ in Eq. (1). For the uninformative detector, the likelihood is constant for $\tilde{\Lambda}<\tilde{\Lambda}_{t}$ and 0 otherwise. Next, consider an actual detection with a different likelihood function that is featureless at scales $\tilde{\Lambda}_{t}^{0}$. In order to obtain $\tilde{\Lambda}_{m}^{0}<\tilde{\Lambda}_{t}^{0}$ for the actual detection, the corresponding likelihood below $\tilde{\Lambda}_{t}^{0}$ would need to increase on average, compared to the uninformative case, or decrease above $\tilde{\Lambda}_{t}^{0}$. Hence low values become more likely by such a measurement, and a twosided credible interval is probably not meaningful.

In order to provide $\tilde{\Lambda}_{t}^{0}$ for GW170817, we still need an estimate for $\tilde{\Lambda}_{t}$. Here we choose $\tilde{\Lambda}_{t} \approx 800$, motivated by the posterior shown in Fig. 11 of [7], which falls off strongly at larger values, indicating that tidal effects of this size can in fact be distinguished. Note the scale $\tilde{\Lambda}_{t}$ could also be lower, which would imply a lower $\tilde{\Lambda}_{t}^{0}$. Since we use $\tilde{\Lambda}_{t}^{0}$ as a conservative sanity check for the interpretation of lower limits, this is not a problem. The case $\tilde{\Lambda}_{m}^{0} \leq \tilde{\Lambda}_{t}^{0}$ can be regarded as a strong indication, but not conclusive evidence, that $\tilde{\Lambda}_{m}^{0}$ is mainly a consequence of the prior assumptions. In this case, providing credible intervals is insufficient and a detailed comparison of posterior and prior shape is necessary.

\section{Universal relations in Bayesian analysis}

We now turn to discuss the use of universal relations in Bayesian analysis of GW signals. To focus on the essential problems, we consider the simplified case where chirp mass and mass ratio are known exactly, but not the tidal effects.

In [10], the tidal effects are encoded in one parameter $\Lambda_{s}$, for which a flat prior distribution in the range $(0,5000)$ is assumed, independent of chirp mass and mass ratio. The prior for the quantity that is actually measured, $\bar{\Lambda}$, is then derived from the universal relations. The result for the given chirp mass and various mass ratios is shown in Fig. 5. As already mentioned, the universal relations have poles that cause gaps and peaks at very small values of $\bar{\Lambda}$. More importantly, the strict prescription of the slope causes gaps extending to larger values of $\bar{\Lambda}$ at certain mass ratios, because otherwise $\Lambda_{1}$ becomes negative. This makes the analysis unsuitable for providing lower limits on $\bar{\Lambda}$ based on measurement.

Further, the upper limit for $\bar{\Lambda}$ depends on the mass ratio. The prior density at fixed $\bar{\Lambda}$ is therefore reduced with increasing mass ratio, as shown in Fig. 5 (the prior for $\tilde{\Lambda}$ shows a similar behavior). This is not a physical assumption, but an artifact of the functional form of the universal relation in conjunction with the choice of a flat prior with constant cutoff for $\Lambda_{s}$. For given $\bar{\Lambda}$ or $\tilde{\Lambda}$, the prior used in [10] favors unequal mass systems, independent of the chirp mass. Therefore we expect this issue to cause a bias away from the equal mass case. We note that the prior for the masses itself already favors unequal mass systems, again not due to physical considerations but due to fixed limits prescribed for the chirp mass.

We conclude that the use of universal relations in Bayesian analysis -in contrast to an ideal measurement discussed in Sec. III A- can also affect the measured quantity $\bar{\Lambda}$, via the prior. However, the prior used in [11] does not exhibit this problem, thanks to an additional cutoff in $\tilde{\Lambda}$ and a simpler choice of universal relations. The prior for $\tilde{\Lambda}$ from [11] is flat, without gaps, and independent of the mass ratio.

The universal relations are used in [10] to derive posteriors for $\Lambda_{1}$ and $\Lambda_{2}$. We note that, for given $q, \Lambda_{1}$ and $\Lambda_{2}$ are uniquely determined by the parameters $\bar{\Lambda}$ and $\bar{S}$. While $\bar{\Lambda}$ enters the GW waveform model, $\bar{S}$ does not and can therefore not be measured at all. All information on $\bar{S}$ is derived from the universal relations. We thus treat it as a statistical variable derived from $\bar{\Lambda}$ based on a model (the universal relations).

The resulting prior distribution for $\bar{S}$ is shown in Fig. 6 . For any mass ratio, the distributions are strongly peaked and asymmetric. The shape of the prior is given entirely by the functional form of the universal relation. It is not related to the distribution of $\bar{S}$ resulting for the EOS models (which would not be meaningful anyway since the EOS selection is arbitrary). For the universal relations in [11], the prior for $\bar{S}$ is even more restrictive, given by a delta function, which is shown as a vertical line in Fig. 6 .

We note that the prior resulting from the universal relations is not used directly in [10]. Instead, the prior for $\Lambda_{a}$ is broadened in order to account for the error of the universal relations, which is estimated from the residuals of the EOS set used for calibration. The broadening of $\Lambda_{a}$ corresponds mainly to a broadening of $\bar{S}$, but to some extent also of $\bar{\Lambda}$. The sharp features visible in Figs. 5 and 6 are smoothed in the final prior used in [10], and the gaps in the prior of $\bar{\Lambda}$ gain some partial support. This might alleviate, but not solve, the issues discussed here. For the rest of this article, we therefore ignore 


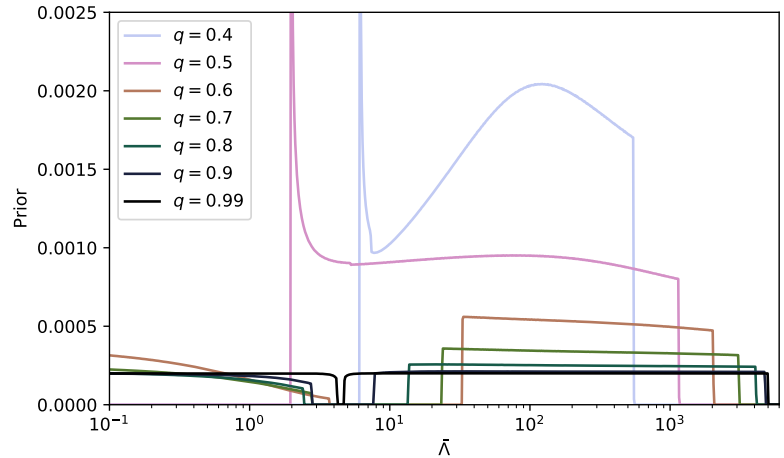

FIG. 5. Prior distribution of $\bar{\Lambda}$ obtained using the universal relation $\Lambda_{a}\left(\Lambda_{s}, q\right)$ with a flat prior for $\Lambda_{s}$ in the range $(0,5000)$, fixed values for the mass ratio, and the given chirp mass. The logarithmic $\mathrm{x}$-axis allows one to see the gaps in the distribution. Note however that the curves show the probability density of $\bar{\Lambda}$, not $\log (\bar{\Lambda})$.

the effects of the additional broadening.

We also note that deriving the prior of $\bar{S}$ from a flat prior of $\Lambda_{a}$ and the universal relations and then broadening it to account for errors of the latter is not the only possible approach. Further, [10] uses error estimates based on treating an arbitrary selection of EOS models as if it were statistical sampling of the true EOS, arriving at a complicated model for the error that depends on $\Lambda_{s}$ and $q$. A cleaner and simpler approach would be to directly prescribe a prior in $\bar{S}$, e.g., a Gaussian, with mean value and broadness defining the model assumptions. That way, one retains control over the shape of the prior distribution and considerably simplifies the interpretation of the assumptions.

The gaps in the priors of $\bar{\Lambda}$ used in [10] in conjunction with the slope $\bar{S}$ restricted by the universal relations have an important consequence for the prior of $\Lambda_{2}$, which is shown in Fig.7 As one can see, it has even larger gaps than the prior for $\bar{\Lambda}$. For mass ratio 0.7 , the gap extends up to $\Lambda_{2} \approx 100$. The method is therefore particularly unsuitable to measure lower limits on $\Lambda_{2}$.

We now apply the uninformative detector test introduced in Sec. III B to the prior used in [10]. As in [10], we use a flat prior in $\Lambda_{s}$, cut off at $\Lambda_{s}=5000$. Following [10], we use the universal relations $\Lambda_{a}\left(\Lambda_{s}, q\right)$ to compute the $\Lambda_{i}$ of the two NS, and further the radii $R_{i}=M_{i} / C_{u}\left(\Lambda_{i}\right)$ (we do not apply the additional broadening of the prior, though). We exclude samples from the prior that violate $\Lambda_{2} \geq \Lambda_{1}>0$. The fifth percentiles of the hypothetical posteriors of the radii that follow from the uninformative detector test are shown in Fig. 8 for a range of mass ratios, as a function of $M_{i}(q)$. As one can see, the radii are still as large as $\approx 8 \mathrm{~km}$ in the mass range of interest.

What is the reason for those surprisingly large bounds from our uninformative hypothetical measurement? One cause is the form of the universal relation $C_{u}(\Lambda)$, which is a second order polynomial in $\log (\Lambda)$. Considering any flat distribution in $\Lambda$, it is not surprising that the 5 th/95th percentiles cover

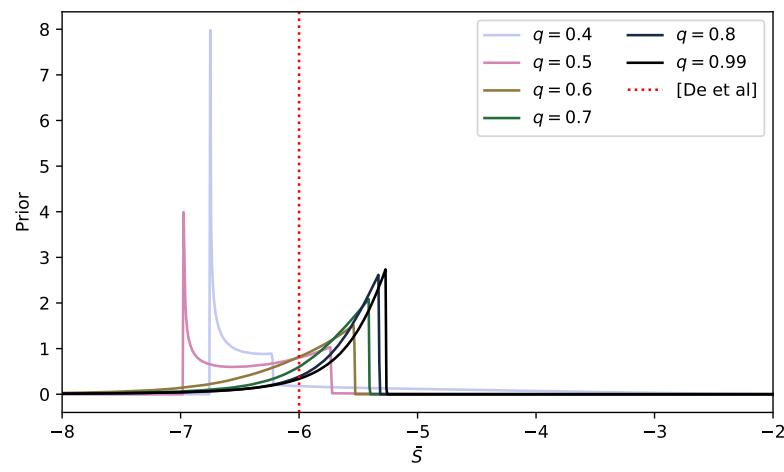

FIG. 6. Like Fig. 5, but showing the prior distribution for the slope $\bar{S}$. In addition, the vertical line represent the unique slope given by the universal relations used in [11].

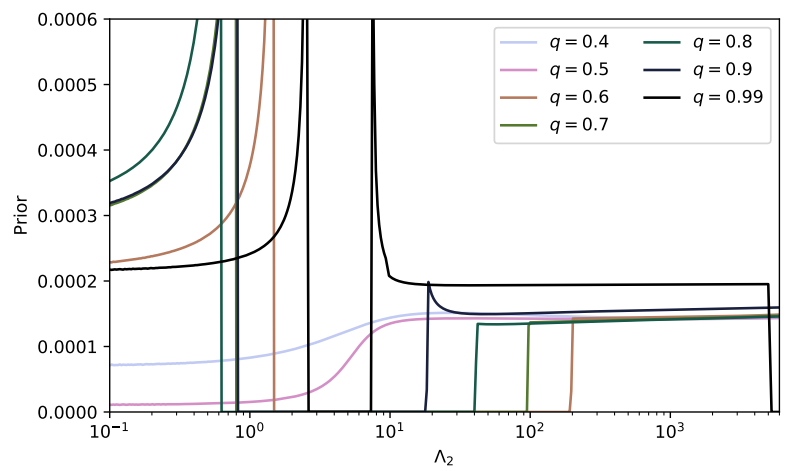

FIG. 7. Like Fig. 5, but showing the prior distribution for the tidal deformability $\Lambda_{2}$ of the lighter NS. Although the x-axis is logarithmic, the curves show the probability density of $\Lambda_{2}$, not $\log \left(\Lambda_{2}\right)$

only a small range of radii. To some extent, the lower limits are also caused by the functional form of the universal relation $\Lambda_{a}\left(\Lambda_{s}, q\right)$, which completely excludes tidal deformabilites below a certain limit (depending strongly on the mass ratio). Translating this exclusion zone to the radius of the lighter NS via $C_{u}(\Lambda)$, we find that radii up to $8 \mathrm{~km}$ are already excluded by construction. This is shown in Fig. 8 as well. It also shows that for extreme mass ratios, further regions in parameter space become unreachable, which is not relevant for the detected event, however, given that the mass ratio is constrained.

The lower limits published in [10] for the measured posteriors are shown as well in Fig. 8 As one can see, the limits are quite similar to those obtained with our hypothetical detector. Comparing the radii derived from the universal relations is not ideal to assess how much the observational data contributed to the posterior. It would be easier to interpret results for the quantity that directly affects the waveform, $\tilde{\Lambda}$. Although [10] do not quote limits for $\tilde{\Lambda}$, they provide $90 \%$ two-sided credible intervals $\Lambda_{1.4}=190_{-120}^{+390}$ for the deformability of a NS with mass $M=1.4 M_{\odot}$, obtained by assuming a linear ex- 
pansion of $\Lambda(M) M^{5}$ around $M=1.4 M_{\odot}$. For comparison, we computed the fifth percentiles of $\bar{\Lambda}$, which increase from 44 at mass ratio $q=1$ to 58 at $q=0.7$, and $\bar{\Lambda} \approx 50$ at $\bar{M}=1.4 M_{\odot}$. The limits from the prior are roughly comparable to the published lower credible bound of 70 . Our findings indicate that the lower radius limits, as obtained in [10] using the universal relations prior, might be largely based on the prior assumptions and less on the observational data. We stress that our qualitative discussion is not sufficient to entirely dismiss the contribution of the data to the lower bounds. Nevertheless, the results denoted in [10] as measurements of lower limits for the radii should not be interpreted as observational evidence for finite size effects yet, even under the assumption that both stars were neutron stars following the same EOS.

A more basic test than our uninformative detector is to visually compare the prior and posterior distributions. Such a comparison is shown in the top-left panel of Fig. 3 of [10]. The prior for the radius of the lighter NS is much less restrictive than the posterior, also towards small radii. This might be interpreted as confirmation that the lower bound is indeed based on data, contrary to our previous discussion. However, there is a subtle but profound pitfall: the priors for the radii shown in [10] are not restricted to the mass range where the posterior has support. In particular, the full prior contains a range of chirp masses. The mass enters directly in the inferred radius when using the compactness-deformability universal relation. In other words, any measurement of the mass already restricts the radius range, even without measuring the deformability.

Comparing the radius posterior to the full prior is therefore not a valid test to assess if the measurement of the deformability is informative. Showing only the full prior of the radii obfuscates the fact that the priors restricted to the almost exactly measured chirp mass are much more restrictive for the inferred radius. In order to judge if the lower credible bound on the radius corresponds to a measurement of finite tidal effects or merely reflects a measurement of the mass range in conjunction with the model assumptions, one should instead compare the radius posterior to the radius prior restricted to measured chirp mass and mass ratio range. A very similar problem was already discussed in the context of spin precession in binary black hole mergers [30, 31].

We carried out the same analysis also for the universal relations from [11]. Similar to the previous discussion, we consider fixed masses and a flat prior for the overall tidal deformability, this time in terms of the parameter $\Lambda_{0}$ used in [11], with the same cutoff at $\Lambda_{0}=5000$. Since [11] remove tidal deformabilities violating limits from causality considerations [29], we apply a cut $\tilde{\Lambda} \leq 51$, derived in [29] for the chirp mass of GW170817 and assuming a maximum NS mass $\geq 2 M_{\odot}$.

As before, we apply our uninformative detector test to obtain a hypothetical posterior. We find that the fifth percentile for the radii are very close to the lower bounds inferred in [11] for the actual detection. This is shown in Fig.9, together with the limits obtained for causal neutron-star models.

Figure 3 in [11] shows the posterior of the effective tidal deformability. The distribution drops sharply to 0 a bit below the lower credible bound. Note, however, that low values of

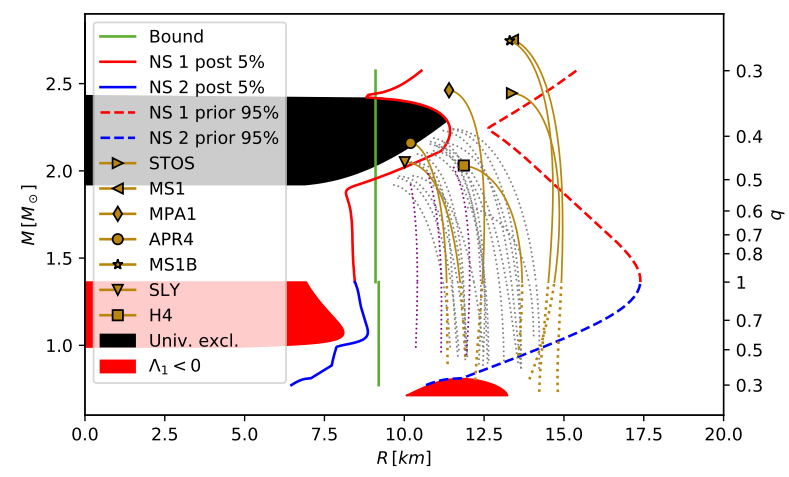

FIG. 8. The solid blue and red curves show the fifth percentile of the NS radii derived from the prior used in [10], restricted to $\tilde{\Lambda}<800$. Those are not boundaries of a two-dimensional distribution; rather the percentiles are computed for each mass ratio $q$ and plotted versus $M_{1}(q)$ (blue) and $M_{2}(q)$ (red). The dashed blue and red curves show the 95th percentiles of the full prior. The black and red areas correspond to values excluded by the universal relations in [10]. The lower credible bounds inferred in [10] for the radii of the two NS using the universal relations are shown as two vertical green lines. For comparison, we also show the mass-radius diagrams for a representative set of nuclear physics EOS models. The curves are cut at the mass of the lighter NS in a binary where the heavier NS mass is maximal (with our example chirp mass).

$\tilde{\Lambda}$ incompatible with causality have been removed from the posterior shown in [11], which likely causes the drop. The limit $\tilde{\Lambda} \leq 51$ from [29] is comparable to the size of the gap. The original posterior and the prior are not shown in comparison, but [11] provide limits both before and after removing causality-violating points from the distribution. The removal increases the lower bounds for $\tilde{\Lambda}$ by $\approx 40$. When using a prior without the causality cut, both the uninformative detector and the analysis of actual data in [11] result in lower limits reduced by a similar amount.

Our findings indicate that the lower credible limits inferred by [11] are almost entirely due to the prior assumptions, while the measurement did not add significant support for finite tidal deformabilities. This calls into question the claim of "first evidence for finite size effects using gravitational-wave observations" [11]. Although [11] compute Bayes factors around 10 in disfavor of $\tilde{\Lambda}<100$ compared to the full range, an erratum [32] reports that the employed thermodynamic integration is unsuitable for computing Bayes factors. The impact on the Bayes factor discussed above is not provided, however.

Besides the problems mentioned before, we also find that the lower limits on radii or tidal deformabilites published in [10, 11] are well below the validity range of the universal relations used in their deviation. Figures 8 and 9 show the limits in comparison to radii obtained for a number of EOS, including those used to calibrate the relations used in [10]. The lower bounds for the radii are the result of evaluating phenomenological expressions in a range explicitly forbidden by the models used to derive the very same expressions (see Sec. II C). 


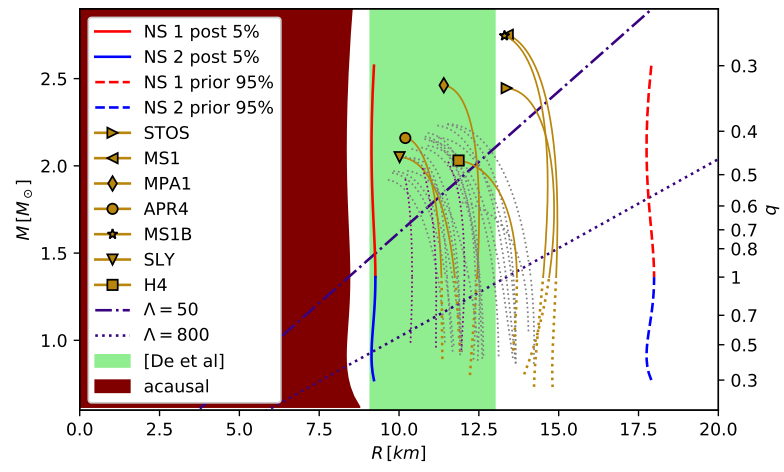

FIG. 9. Like Fig. 8 but for the universal relations from [11]. The green shaded area marks the statistical bounds reported for the "common radius" in [11], not including the systematic errors, and based on a prior that excludes tidal deformabilities below the causality limit. The brown shaded area shows causality-violating region obtained by combining the bound $\tilde{\Lambda} \leq 51$ derived in [29] and the universal relations from [11]. For comparison, we also show the radii derived using $C_{u}$ (the version from [11]) for fixed values of $\Lambda$.

\section{EOS-agnostic Bayesian analysis}

In this section, we discuss the analysis of tidal effects in [7], with a focus on the interpretation of lower limits. As in previous sections, we use the idealized case of known masses and zero spins to highlight problems that are not obvious in the full statistical analysis.

In [7] it is not assumed that both stars obey the same EOS, or that they are neutron stars, and no universal relations are employed. In detail, [7] employs a flat prior for the tidal deformabilities $\Lambda_{1}, \Lambda_{2}$, cut at a maximum value $0 \leq \Lambda_{1}, \Lambda_{2}<$ 5000. This implies that the tidal deformabilities are not only allowed to differ for equal mass systems, but also that they are completely uncorrelated, i.e. it is not assumed that stars with similar mass are more likely to have similar deformabilities.

The assumptions of uncorrelated deformabilities have an important consequence for the prior of $\tilde{\Lambda}$. Because of the rectangular uniform support in $\left(\Lambda_{1}, \Lambda_{2}\right)$ space, it linearly approaches 0 near $\tilde{\Lambda}=0$, with a slope that depends on the mass ratio. Figure 10 shows the prior resulting for $\tilde{\Lambda}$ restricted to various mass ratios. Small values of $\tilde{\Lambda}$ are already disfavored by the prior distribution. Obviously, this needs to be considered before interpreting lower credible bounds as a proof of finite size effects.

In [7], the raw posterior of $\tilde{\Lambda}$ is divided by the marginalized prior of $\tilde{\Lambda}$ before computing credible intervals on $\tilde{\Lambda}$. This approach effectively corresponds to using a prior in $\tilde{\Lambda}$ that is flat after marginalization over mass ratio. The motivation given in [7] is to address the issue above, although the physical motivation for the modified prior is not explained. Since the behavior of the original prior is a direct consequence of the assumption of uncorrelated deformabilities, scaling it to obtain a flat prior in $\tilde{\Lambda}$ implies that the posterior obtained for $\tilde{\Lambda}$ is based on contrary assumptions. We note that the new prior does not cor-

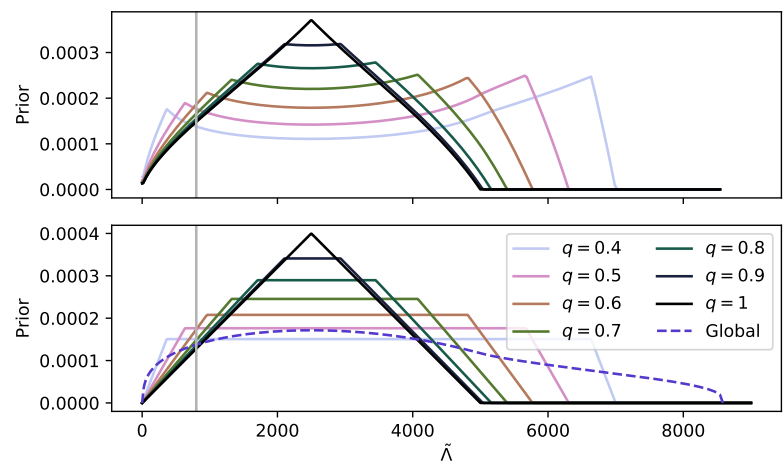

FIG. 10. Bottom: Prior distribution of effective tidal deformability $\tilde{\Lambda}$ derived from the EOS-agnostic flat $\Lambda_{1}, \Lambda_{2}$ prior. The dashed curve shows the full prior marginalized over all mass ratios; the other curves show the prior restricted to various mass ratios. Top: modified prior distribution for $\tilde{\Lambda}$ obtained by dividing the original prior by the prior after marginalizing out the mass ratio. The different curves show the modified prior restricted to the same mass ratios as the bottom panel. We note the prior density at $\tilde{\Lambda}=0$ is small, but not 0 .

respond to the assumption of a common EOS, since the NSs can still have the same masses but different deformabilities. However, in this work we are not arguing what prior should be used, but how much the ones used determine the results.

First, we investigate more carefully the technical implications of the rescaling. One problem is that scaling in a mass ratio independent way is not the only choice to obtain a flat marginalized prior. This already introduces an ambiguity that has not been discussed. More importantly, the resulting prior restricted to mass ratios relevant for the detected event is not flat at all. The reason is that the prior for the mass ratio strongly favors unequal mass systems (mainly due to the limits imposed on the chirp mass) for which the prior of $\tilde{\Lambda}$ has increasingly large slope near $\tilde{\Lambda}=0$. For moderate mass ratios, the reweighted prior used in [7] disfavors low values of $\tilde{\Lambda}$, despite the reweighting procedure. This is shown in the top panel of Fig. 10 .

To asses the contribution of the prior to the lower limits we now apply the uninformative detector test introduced in Sec. III C to the reweighted prior used in [7]. We obtain a lower fifth percentile of $\tilde{\Lambda}=128$ for the posterior, for all mass ratios $q \gtrsim 0.6$. This should be compared to the fifth percentile of the (reweighted) posterior inferred from the actual data in [7] for the low-spin prior, which is $\tilde{\Lambda}=110$ (the $90 \%$ credible bound of the mass ratio is $q>0.73$ ). Under the assumption of low spins, [7] interprets the credible lower bound as evidence for finite tidal effects. In contrast, our discussion strongly indicates that the lower bound should be regarded mainly as a consequence of prior assumptions in conjunction with the observational constraints on the mass ratio.

In the remainder of this section, we compare the EOS agnostic approach to the studies based on universal relations. Surprisingly, the results in [7] can be reinterpreted under the assumption that both objects are neutron stars obeying the 
same EOS, in the sense that the tidal deformability is a unique but unknown function of mass. For this, one only has to exclude systems with mass ratio exactly 1 , which makes no difference because this set has infinitesimal support in parameter space. Further, one has to allow any functional form of the tidal deformability $\Lambda(M)$, including arbitrary steep gradients. In our notation, this implies an unbound range $-\infty \leq \bar{S} \leq \infty$. Note all EOS considered here fulfill $\bar{S}<0$.

Figure 11 illustrates the the prior distribution of the slope $\bar{S}$ for fixed chirp mass and various fixed mass ratios. As expected, the support increases towards positively and negatively infinite slopes when approaching equal mass. We recall that Eq. (12) then implies $\mathrm{d} \Lambda / \mathrm{d} M \rightarrow \pm \infty$. At the same time, the prior probability for any finite slope range approaches 0 in the equal mass limit. For unequal masses, the prior support for a given range can also increase with increasing $q$. In any case, there is a strong dependence on mass ratio.

Since the signal is (almost) independent of the slope, the slope posterior is given by the prior. Compared to a slope prior bounded to some model-dependent range, the posterior for quantities derived from the slope, such as the individual tidal deformabilities, is broadened. Compared to a more plausible prior $\bar{S}<0$, on can also expect a strong bias of $\Lambda_{1}$ and $\Lambda_{2}$ towards larger and smaller values, respectively.

For comparison, we also plot the range of $\bar{S}$ obtained from our standard set of EOS. Comparing also to Fig. 6, one can see that the EOS-agnostic study and the studies based on universal relations occupy opposing ends of a spectrum of assumptions. The EOS-agnostic study is overly inclusive regarding the slope, compared to the range of theoretical EOS models, while the universal relations strongly restrict the slope. The most restricted slope is given by the fixed value used in [11], while [10] further broadens the raw prior distribution resulting from their universal relations to account for a certain amount of systematic error. Given that the universal relation assumptions in [10] strongly restrain $\bar{S}$, it is hardly surprising that the credible regions reported occupy a smaller volume in parameter space.

\section{E. Parametrized EOS approach}

A complementary approach [10] to EOS-agnostic and universal relation methods is based on parametrizing the EOS and including the corresponding parameters in the statistical analysis. This approach is also slightly problematic, as we explain in the following. Imagine an observation where chirp mass and mass ratio are measured exactly, but for $\bar{\Lambda}$ only a probability distribution is known. Further, consider a hypothetical EOS parametrization where one parameter $P_{u}$ uniquely determines $\bar{\Lambda}$ for the measured masses, while the remaining parameters $P_{i}$ have no influence on $\bar{\Lambda}$, but do have an impact on the individual NS properties. Since the parameters $P_{i}$ have no influence on the measured quantity, their posterior distribution is given by the prior assumed for $P_{i}$. Therefore, the posteriors for individual NS properties depend on the priors for $P_{i}$. For the parametrization used in [10], the parameters are not as cleanly separated, which makes it difficult to assess the im-

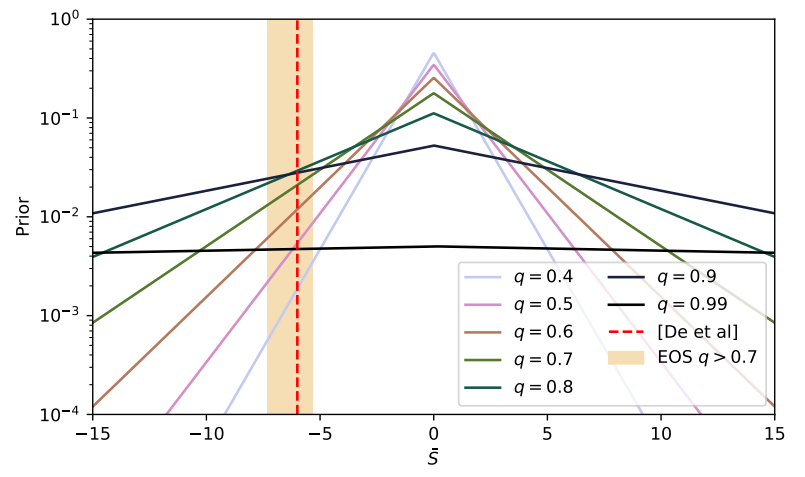

FIG. 11. Prior distribution of the slope parameter $\bar{S}$ resulting from a flat prior in $\Lambda_{1}, \Lambda_{2}$ space, restricted to $0 \leq \Lambda_{i} \leq 5000$, as used in [7]. We show priors for various fixed mass ratios. Although we only show a restricted range, the prior support extends to $\pm \infty$. The shaded band marks the range spanned by our standard EOS set for mass ranges $q>0.7$. The dashed line marks the value $\bar{S}$ corresponding to the universal relations used in [11].

portance of the above. Note that the implicitly chosen prior in the parameters $P_{i}$ takes over the role of the analytic prescriptions for $S_{m}$ in the universal relation method when it comes to inferring individual NS properties. Knowledge of the priors for a parametrization $P_{i}, P_{u}$ instead of the ones reported in [10] is required to interpret the significance of single-NS tidal deformabilities and radii inferred with the parametrized EOS method.

Since there is no natural choice for the exact parametrization and no other experimental data were used, the shape of the prior distribution should be regarded as an arbitrary choice. It is therefore important to assess how much the prior contributes to any results derived from the posterior.

The prior and posterior for the radii are compared in the topright panel of Fig. 3 in [10]. The credible bounds for the prior distribution are not provided. Visually, it seems that the prior already has little support below the published lower limits of the posterior. In addition, the comparison is complicated by the fact that the full prior is shown, not restricted to the measured mass range (compare the discussion in Sec. IIIC). It is therefore impossible to judge how much of the lower limit is based on a measurement of the masses in conjunction with the prescribed prior for the EOS parameters, and how much on an actual imprint of tidal effects in the data.

We note that instead of parametrizing the EOS, one could directly parametrize the function $\Lambda(M)$, for example, using a polynomial. One can then perform Bayesian parameter estimation of the corresponding coefficients. This approach was suggested in an earlier work [33]. When considering a single ideal measurement, an obvious problem arises. As discussed earlier, a single ideal detection provides one data point $\bar{\Lambda}, \bar{M}$. This single point cannot determine more than one coefficient, or combination of coefficients. For example, if $q \approx 1$, then $\bar{\Lambda}$ determines $\Lambda(M)$ at $M=\bar{M}$. The slope of $\Lambda(M)$ at $M=\bar{M}$ on the other hand depends on $\bar{S}$, which cannot be 
measured. Higher derivatives also have no impact on the measured signal. For unequal mass systems, the system is equally underdetermined and one can only measure one combination of coefficients. Bayesian parameter estimation nevertheless yields posteriors for all the coefficients, and some might appear to be constrained by measurement. However, the signal still only carries one degree of freedom regarding the coefficients. We recall that the shape of the posterior distribution in $\bar{\Lambda}, \bar{M}$ around the true value has nothing to do with the behavior of the $\Lambda(M)$ curve, but rather reflects the detector noise and the relative impact of mass ratio and deformability. It cannot be used to constrain the slope of $\Lambda(M)$, for example.

The approach is also instructive regarding the parametrized EOS method, which in essence also contains a parametrization of $\Lambda(M)$, but adds further degrees of freedom that leave the curve $\Lambda(M)$ invariant. We note, however, that the requirement of causality imposes constraints on the EOS. Assuming there is only one branch of solutions, and not a transition to quark stars, for example, this implies constraints on $\Lambda(M)$. Results about $\Lambda(M)$ that have no impact on the information in the signal, such as $\bar{S}$, are not completely arbitrary. It is however very difficult to disentangle the meaningful constraints from the results of Bayesian analysis employing the parametrized EOS.

\section{F. Systematic errors of EOS constraints}

In the following, we discuss how systematic errors of the models for gravitational signals affect constraints of the tidal deformability that would result from a perfect detection (in the sense of arbitrary large signal-to-noise ratio) of the inspiral phase. Naturally, the impact of waveform systematics in statistical analysis of actual measurements with noisy data cannot be any smaller.

In the sensitive frequency range of current detectors, gravitational waveforms of coalescing NSs can only be computed using semianalytic approximations. Although some models are calibrated with numerical relativity simulations, we note that such simulations cover only the upper end of the sensitive frequency range of current detectors (in contrast to the $\mathrm{BBH}$ case, where the merger typically occurs at lower frequencies).

Currently, the error of these models can only be estimated by comparing waveforms based on different approximations. In Bayesian statistical data analysis using matched filtering, the systematic error of the waveform model causes a corresponding bias of the posterior probability distributions. It is worth pointing out that the bias of tidal effects is not simply determined by the accuracy with which the tidal effects are modeled, but is also sensitive to the accuracy of the underlying waveform model for zero deformability (binary black hole case).

A central measure in $\mathrm{GW}$ analysis is the mismatch between waveforms. The likelihood $P(d \mid \theta, I)$ in Eq. (1) depends directly on the mismatch between waveform model and data. It expresses how much waveforms differ from the perspective of a given detector, weighting different frequency components by detector sensitivity:

$$
F\left(h_{1}, h_{2}\right)=1-\max _{\phi_{c}, t_{c}} \frac{\left(h_{1}^{\phi_{c}, t_{c}}, h_{2}\right)}{\sqrt{\left(h_{1}, h_{1}\right)\left(h_{2}, h_{2}\right)}}
$$

where $h_{1}^{\phi_{c}, t_{c}}$ denotes waveform $h_{1}$ shifted in time and phase by $t_{c}$ and $\phi_{c}$, and

$$
\left(h_{1}, h_{2}\right)=4 \Re\left(\int_{f_{\min }}^{f_{\max }} \frac{\tilde{h}_{1}(f) \tilde{h}_{2}^{*}(f)}{S_{n}(f)} \mathrm{d} f\right),
$$

where a tilde denotes Fourier transform and $S_{n}$ is the detector spectral noise density. In the following we use the advanced LIGO zero-detuned high-power design sensitivity curve (provided by the LALSUITE [34] software library, see also [35]) and apply a high-frequency cutoff at $f_{\max }=1 \mathrm{kHz}$ when computing waveform mismatches.

Although we discuss the case of arbitrary loud signals, the results in this section are still specific to LIGO-type detectors. They do not depend on the overall sensitivity though, only the relative sensitivity at different frequencies. Further, the choice of cutoff parameter does have a varying impact on the estimates we will present. Since the cutoff frequency in [7] is chosen dynamically using heuristic criteria based on masses and tidal parameters, the exact impact of the waveform systematics might be somewhat larger or smaller than our estimates for fixed cutoff. We will not address this additional complication here, but note that it deserves consideration before claiming observational evidence for tidal effects.

Before discussing the impact of waveform systematics in general, we start with a more specific question. When computing a waveform with one waveform model evaluated at some example parameters, and computing the best match with a different waveform model, how strongly do the parameters differ? We focus on the idealized case of fixed chirp mass (using the GW170817 value) and zero spin, working in a parameter space $\bar{M}, \bar{\Lambda}$. Figure 12 shows the best matches with regards to a few reference parameters, comparing the IMRPhenomD_NRTidal [36] and TaylorF2(6PN) [37] waveform models. We note that the two do not share the same pointparticle baseline and that the TaylorF2 shows relatively large differences to more recent models (see also [7]). How the true error of the most sophisticated models compares to the differences found for this example pair is an important open question, but outside the scope of this work.

As one can see, the differences for reference points with zero deformability are comparable to the lower credible bounds for the tidal deformability given in [7] (with narrow spin prior). In contrast, the Bayesian lower bounds obtained in [7] for different waveform models (including the two examples used here) agree quite well. This is not necessarily a contradiction. As we discussed before, one would expect similar lower bounds even when low tidal deformabilities cannot be distinguished at all at the given sensitivity. The comparison of statistical parameter estimation studies with different waveform models is an important cross-check, but it should not be used exclusively to estimate the systematic error due to the waveform. On the contrary, if lower bounds agree better 
than the uncertainties obtained from the measures discussed here, it should be taken as an indication that the lower bounds are not informative.

The comparison of best matches for different waveform models gives a reasonable lower limit for the waveformrelated systematic parameter uncertainty. We note, however, that the parameter difference at the best match could a priori be 0 despite a large residual mismatch at the best matching parameter. Further, true waveform most likely differs from both models.

We therefore devised a complementary error estimate that is not based on the best matching parameters, but on the magnitude of mismatch. For this, we consider two waveform models $A, B$ and two points $P_{1}, P_{2}$ in parameter space. The mismatch between waveform model $A$ evaluated at the two points can be used as a measure of how mismatch changes with distance in parameter space. The mismatch between waveform models $A$ and $B$ evaluated at the same point $P_{1}$ can be used to give us a scale for mismatches between waveform models. Comparing the two, we define a distance in parameter space that is symmetric in the two waveform models,

$$
D\left(P_{1}, P_{2}\right)=\frac{\min \left(F\left(a_{1}, a_{2}\right), F\left(b_{1}, b_{2}\right)\right)}{\max \left(F\left(a_{1}, b_{1}\right), F\left(a_{2}, b_{2}\right)\right)},
$$

where $a_{i}$ and $b_{i}$ are the waveforms obtained with models $A$, $B$ at points $P_{i}$.

If two parameter sets differ by less than $D<1$, they cannot be distinguished reliably by measurement as long it is not known if $A$ or $B$ is the correct model. In practice, we have to assume that the true systematic error is bounded below $D<E$ if $D$ is computed for a pair of actual waveform models, where $E$ is a guess of how much larger the true error is compared to the differences between available models.

The contours $E=1,2$ are shown in Fig. 12, again for IMRPhenomD_NRTidal and TaylorF2(6PN). It is worth noting that for reference points with zero deformability, the differences to the best matching parameters are much smaller than the extent of the $D=1$ contours. This might indicate that the mismatch changes only slowly for low tidal deformability. The slower the change in mismatch, the larger the errors of the deformability. The location of the best match is probably not a good error estimate in this case. This concerns studies like [17], which use the best match location to estimate systematic errors, and which might similarly underestimate the true parameter error.

As a more conservative error estimate, we can use the maximum of the two heuristic measures. From this, we conclude that for mass ratios in the range $0.7 \leq q \leq 1$ and fixed chirp masses around the one for GW170817, we cannot distinguish a deformability $\bar{\Lambda} \approx 200$ from the binary black hole case $\bar{\Lambda}=0$, unless the waveform model employed is known to be more accurate than the difference between the IMRPhenomD_NRTidal and TaylorF2 models, i.e. $E<1$.

For comparison, [11] claims evidence for finite-size effects based on lower limits $\tilde{\Lambda}>38,54,45$ obtained for the three different priors considered therein (those limits are the ones obtained without applying a manual lower cutoff motivated

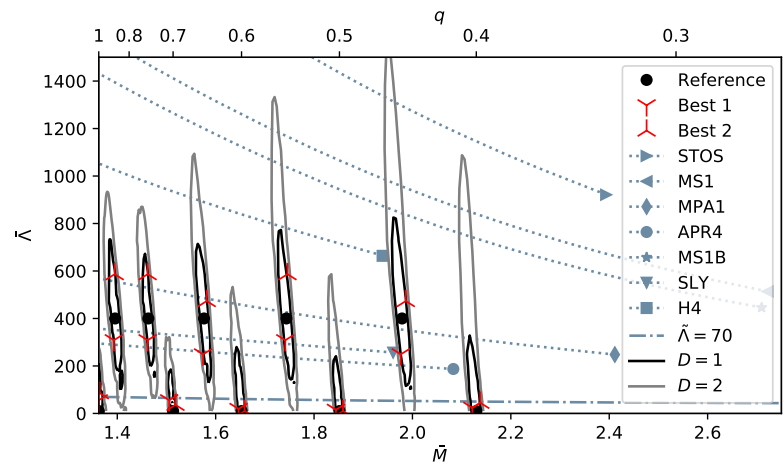

FIG. 12. Systematic error of $\bar{\Lambda}, \bar{M}$ due to BNS waveform modeling, with respect to selected reference points (black circles). The error is estimated with two measures. The contours show $D=1$ (black) and $D=2$ (grey) according to Eq. 18 based on comparing IMRPhenomD_NRTidal and TaylorF2(6PN) waveform models. The markers labeled "Best 1" and "Best 2" show the location of the best match for each of the models to the other one evaluated at the reference point. For comparison, we also show the curves obtained for different EOS in $\bar{\Lambda}, \bar{M}$ space, and the HPD bound $\tilde{\Lambda}>70$ from [7].

by causality constraints in NS models). In light of our waveform systematic discussion, this claim implies a great deal of trust in the TaylorF2 waveform model used in [11]. Similar arguments apply to [10], which does not provide a limit for $\tilde{\Lambda}$, but lower limits for the individual radii. Using the same universal compactness relations as [10], we find that $\bar{\Lambda}=200$ corresponds to a NS radius of $10.4 \mathrm{~km}$ for the equal mass case, which is again larger than the lower limit $R>9.1 \mathrm{~km}$ given in [10].

\section{G. Improving GW analysis}

In the following, we propose an alternative to the universal relation and EOS-agnostic analysis methods that avoids the shortcomings discussed in Secs. III A III C

The first ingredient is to prescribe the priors for $\bar{\Lambda}$ and $\bar{S}$ directly. In contrast to the universal-relation-based parametrization used in [10], one retains direct control over the $\bar{\Lambda}$ prior, avoiding also the gaps discussed in Sec. III C. It also avoids correlation with the prior for the mass ratio. One can simply prescribe a flat prior for $\bar{\Lambda}$. This avoids disfavoring low values of $\bar{\Lambda}$ prior, contrary to the EOS-agnostic flat $\Lambda_{1}, \Lambda_{2}$ prior. We stress that this flat prior is still an ad-hoc choice not based on any independent observations. One should always check how much results depend on such a prior.

When using $\bar{\Lambda}$ and $\bar{S}$ as independent variables, the individual deformabilities are given by

$$
\Lambda_{1}=q^{-\bar{S} / 2} \bar{\Lambda}_{0}, \quad \Lambda_{2}=q^{\bar{S} / 2} \bar{\Lambda}_{0},
$$

where

$$
\bar{\Lambda}_{0}=\frac{\bar{\Lambda}}{w_{1}(q) q^{-\bar{S} / 2}+w_{2}(q) q^{\bar{S} / 2}} .
$$


The upper cutoff for the $\bar{\Lambda}$ prior can be set to any reasonable cutoff for the tidal deformability of a single NS. From Eqs. (5) and $\sqrt{6}$ it is easy to show that $\bar{\Lambda} \leq \max \left(\Lambda_{1}, \Lambda_{2}\right)$ for all mass ratios.

The main advantage of specifying the prior directly for $\bar{\Lambda}$ is that the prior for the second parameter (here $\bar{S}$ ) does not influence the posterior of $\bar{\Lambda}$. The reason is that $\bar{\Lambda}$ is the only relevant parameter for the GW signal, unless $\bar{S}$ reaches values that would cause significant corrections in the waveform model employed. Although the corrections may never become significant unless the signal-to-noise ratio is much better than GW170817, this needs to be validated in any careful analysis. We propose performing parameter estimation with a prior in $\bar{S}$ that is much wider than any model assumptions one intends to make. Most likely, it will turn out that various model assumptions of interest constrain $\bar{S}$ much more than the observational data (at least for any detection comparable to GW170817). This simplifies the parameter estimation task considerably because model assumptions for $\bar{S}$ do not need to be included during the costly parameter estimation computations, but can be incorporated in a postprocessing step, as we detail later.

The second ingredient is to express EOS constraints inferred from the measurement in terms of credible regions in the parameter space $M_{c}, \bar{M}, \bar{\Lambda}$. Compatibility with a given EOS can be tested by comparing to the two-dimensional subspace allowed by the EOS. For a detection similar to GW170817, the chirp mass is known very accurately and can be treated as fixed. In that case, the EOS surface reduces to one-dimensional curves in $(\bar{M}, \bar{\Lambda})$ space, as in Fig. 11. This approach also allows comparing to the mixed binary hypothesis with given EOS in a unified way, without resorting to separate parameter estimation studies with parameter spaces of different dimensionality, or to the EOS-agnostic method. It also allows one to judge the compatibility of arbitrary EOS models without new parameter estimation computations.

One should avoid providing results from GW data primarily in terms of Bayesian credible regions on the NS mass-radius relations. Although it simplifies comparison to astronomical observations of isolated pulsars and allows one to use known mass-radius curves for EOS models, it also requires additional model-dependent assumptions, e.g., universal relations. As we have seen, it is nontrivial to assess how much the final result depends on measurement and how much on model assumptions. In contrast, $M_{c}, \bar{M}$, and $\bar{\Lambda}$ are directly constrained by the GW signal and do not require model assumptions. Presenting results in this parameter space seems the cleanest line of separation between the overlapping problems of GW data analysis on one side and NS structure models on the other.

When results on individual NS properties are presented, the unavoidable direct dependency on model-specific constraints of $\bar{S}$ needs to be quantified. Otherwise, it is impossible to judge how much results depend on assumptions and how much on measurement constraints. We note that current models only yield approximate ranges for $\bar{S}$, possibly as a function $M_{c}, \bar{M}$, and $\bar{\Lambda}$, but not a well-motivated shape of the prior distribution. As we remarked earlier, $\bar{S}$ is typically much more constrained by assumption than by measurement. It is therefore not meaningful to repeat expensive parameter estimation computations with different priors for $\bar{S}$. Instead, one can apply cuts to the posterior, limiting $\bar{S}$ to the range allowed by a given model assumption.

To get an estimate on the impact of model assumptions constraining $\bar{S}$ to a range, on can further marginalize the restricted posterior over $\bar{S}$, and then derive two new posteriors for each of the individual deformabilities $\Lambda_{1}, \Lambda_{2}$ by setting $\bar{S}$ to the extreme values allowed by the model constraints. Repeating this for different models provides a computationally inexpensive way to separate model uncertainties and measurement error.

What type of credible intervals, e.g., two-sided symmetric, highest posterior density (HPD), or upper limits, are meaningful to provide for the statistical error depends on the posterior shape. For the tidal deformability, there likely exists a reliable one-sided upper limit, since larger tidal effects are increasingly easier to detect. For a detection similar to GW170817, lower limits require more care, as argued in previous sections. We note that using a HPD interval, as done in [7], does not automatically yield a meaningful choice. For example, after adding an infinitesimal perturbation to a flat posterior, the HPD choice can result in anything from one-sided lower to one-sided upper limits.

In general, one should compare the posterior and prior in detail, also distinguishing the impact of mass constraints and deformability constraints. This is simpler when using a flat prior in $\bar{\Lambda}$ as proposed, since the latter is not correlated with the masses. It is then not necessary to restrict the prior to the measured mass range for the comparison, in contrast to the universal relation method (see discussion in Sec. III C).

A simple diagnostic check is given by the uninformative detector test introduced in Sec. IIIC The cutoff value 800, motivated by GW170817, should of course be replaced with the one-sided upper limit for the posterior at hand. Lower bounds should be interpreted with care if the lower bound is not larger than the one given by the uninformative detector test. In such cases, lower bounds should not be regarded as observational constraints, at least not without a more careful analysis. Even when passing this test, one should still perform Bayesian model selection as in [16], since credible intervals do not imply statements on the likelihood of specific models (see Sec.[I).

The waveform systematics should not be assessed solely by comparing parameter estimation results using different waveforms. It is possible that statistical bounds agree much better than the systematic uncertainty, if the bounds are determined mainly by the prior. One should use mismatch computations as shown in Sec. III F as a guideline, and not trust lower limits below values for which the waveform differences are potentially larger.

We conclude with some remarks on incorporating EM counterparts. Improvements in numerical modeling of counterparts likely allows one to obtain robust predictions of EM counterparts. However, there is no reason to assume that the parameter combinations that are most constrained by EM counterparts will bear any similarity to those combinations constrained by the GW signal. In particular, it might not be possible to express the impact of the EOS on the kilonova signal solely in terms of tidal deformability. In [38], a few nu- 
merical simulations were used to propose a loose correlation between effective tidal deformability and an upper limit for mass ejection. A later work [39] considered different EOS and found counterexamples, also demonstrating a large impact of the mass ratio. For reliable results, it is likely necessary to consistently use a parameter space with enough parameters to uniquely characterize both EM and GW observable signals. One way to construct such a space is to use a parametrized EOS both for GW data analysis and numerical simulations predicting EM counterparts (the latter also requires one to parametrize the thermal part). Since numerical simulations are computationally expensive, analytic fits cannot be avoided completely. In order to prevent conceptual problems similar to those we discussed for the universal relations, they should however be based on data points in the parameter region relevant for a given event.

\section{SUMMARY}

In this work, we investigated the question whether results presented in [7, 10, 11] could be interpreted as observational evidence for tidal effects during the merger that caused gravitational-wave event GW170817. Such a claim was made in [11], and also in [7] assuming low NS spins. Under the assumption that both objects are NSs, the authors of Ref. [10] claim a measurement of lower bounds for the NS radii. Since those are derived by feeding the tidal deformabilities into a given monotonic analytic expression, this claim implies a measurement of lower limits for tidal effects.

All of those works use Bayesian statistics, but do not satisfactorily address the question of how much the results are already determined by the prior assumptions, and how much the observational data contribute. We discussed the prior probability distributions in detail. Their features are based largely on arbitrary choices, which is particularly problematic for lower limits on tidal effects, since small tidal effects have a weaker impact on the measured signal.

Since the detector cannot distinguish small tidal effects as well as large ones, we discussed the idealized case of a detector that cannot distinguish tidal effects at all if they are below published upper credible bounds. Using such manifestly uninformative hypothetical measurement with the same prior assumptions, we still obtained lower limits comparable to the published ones. Our findings indicate that the Bayesian lower credible bounds in [7, 10, 11] might largely reflect prior assumptions. They should not be regarded as observational evidence, at least not without a more detailed analysis.

During our study, we also found several technical and conceptual problems. The two studies [10, 11] are using model assumptions based on universal relations in a way that is not self-consistent. In short, those relations already imply much larger lower limits just based on the measured mass range, and hence cannot be used to measure finite tidal effects. While the use of universal relations in [11] has no consistent motivation when applied to low tidal deformabilties for the mass range of GW170817, it still allows such values. The universal relations used in [10] on the other hand completely exclude values be- low a limit depending on mass ratio. This limit is not a physical assumption, but results from extrapolating a complicated fit function given by a fraction of polynominals.

Regarding the alternative approach of parametrized EOS discussed in [10], we presented arguments suggesting that the impact of the prior is not sufficiently studied to claim observational evidence. However, our discussion is not conclusive since we did not quantify the impact.

Our reassessment of the EOS-agnostic study [7] also revealed some problems. On the conceptual level, we argued that the use of a rescaled posterior, which mimics a flat prior in the effective tidal deformability, in effect corresponds to a prior that not based on well-defined physical assumptions. Moreover, it is ambiguous how the prior in the multidimensional parameter space should be rescaled to achieve a given marginalized prior. On the technical level, we investigated the correlation with the mass ratio and found that the full rescaled prior from [7] is not flat at all when restricted to the measured mass range, defeating the apparent purpose of the procedure.

For use in future studies, we sketched a GW data analysis strategy that sidesteps some of the emerging problems. The main source of complications seems to be the inclusion of quantities that cannot be measured, necessitating the use of model assumptions, and complicating the interpretation of results considerably. The main idea of our proposal is to restrict the data analysis to quantities that actually affect the waveform and can hence be measured. Compatibility with model assumptions can be tested in a second step, as well as derivation of quantities that require further model assumptions. Our strategy also simplifies quantifying the impact of other model assumptions independently.

We demonstrated explicitly how to test compatibility with EOS models. This also showed that, based on a single event with masses both compatible with NS, it is fundamentally impossible to distinguish a BNS merger with one EOS from a BH-NS merger with a different EOS, as long as tidal effects enter the waveform model only via the effective deformability. We also provided examples demonstrating that the maximum mass of NSs cannot be inferred from the inspiral GW signal alone.

Another aspect particularly important for measuring finite tidal effects is the impact of systematic waveform model uncertainty. This was addressed in [7] by repeating the full statistical analysis with different waveform models. A similar approach is to perform parameter estimation studies based on one waveform model on artificial data constructed using a different waveform model. We argued that this is only a valid error estimate for credible bounds if they depend on the observational data, instead of the prior. We derived an independent estimate by directly computing differences between waveforms, using the mismatch measure relevant for $\mathrm{GW}$ data analysis for LIGO-type detectors. The results from this estimate indicate that also previous error estimates based on bestmatching waveforms, e.g., [17], might be too optimistic. We find that the uncertainties expected from differences of current waveform models are larger than the lower limits inferred for GW170817.

Conclusive observational evidence of finite tidal effect 
therefore requires better knowledge of the waveform accuracy, as well as another merger event with either a larger signal-tonoise ratio or involving objects with larger deformabilties. For standard NS models, that means less massive NS. However, the mass range of observed NS is rather limited so it is not guaranteed that such an event will occur.

\section{ACKNOWLEDGMENTS}

We thank Soumi De, Duncan A. Brown, and Katerina Chatziioannou for useful discussions, and Philippe Landry for a careful review of the initial draft of this paper. This work was supported by the Max Planck Society's Independent Research Group Programme. In this work, we use a neutron-star model database computed on the Holodeck cluster at the Albert Einstein Insitute in Hannover.
[1] B. P. Abbott, R. Abbott, et al. (LIGO Scientific Collaboration and Virgo Collaboration), Phys. Rev. Lett. 119, 161101 (2017)

[2] B. P. Abbott et al., Astrophys. J. 848, L13 (2017)

[3] B. P. Abbott et al., Astrophys. J. 848, L12 (2017)

[4] E. E. Flanagan and T. Hinderer, Phys. Rev. D 77, 021502 (2008)

[5] B. P. Abbott et al., Classical and Quantum Gravity 32, 074001 (2015)

[6] F. Acernese, M. Agathos, K. Agatsuma, D. Aisa, N. Allemandou, A. Allocca, J. Amarni, P. Astone, G. Balestri, G. Ballardin, et al., Classical and Quantum Gravity 32, 024001 (2015)

[7] B. P. Abbott, R. Abbott, T. D. Abbott, F. Acernese, et al. (LIGO Scientific Collaboration and Virgo Collaboration), Phys. Rev. X 9, 011001 (2019)

[8] B. P. Abbott et al., Astrophys. J. 851, L16 (2017)

[9] B. P. Abbott et al., Astrophys. J. 875, 160 (2019)

[10] B. P. Abbott, R. Abbott, T. D. Abbott, F. Acernese, K. Ackley, C. Adams, et al. (LIGO Scientific Collaboration and Virgo Collaboration), Phys. Rev. Lett. 121, 161101 (2018)

[11] S. De, D. Finstad, J. M. Lattimer, D. A. Brown, E. Berger, and C. M. Biwer, Phys. Rev. Lett. 121, 091102 (2018)

[12] P. Landry and R. Essick, Phys. Rev. D 99, 084049 (2019).

[13] C. D. Capano, I. Tews, S. M. Brown, B. Margalit, S. De, S. Kumar, D. A. Brown, B. Krishnan, and S. Reddy, (2019).

[14] J. Veitch and A. Vecchio, Phys. Rev. D 81, 062003 (2010)

[15] K. Yagi and N. Yunes, Classical and Quantum Gravity 33, 13LT01 (2016)

[16] B. P. Abbott et al., arxiv:1908.01012 (2019)

[17] M. Favata, Phys. Rev. Lett. 112, 101101 (2014)

[18] S. E. Gralla, Classical and Quantum Gravity 35, 085002 (2018)

[19] K. Chatziioannou, C.-J. Haster, and A. Zimmerman, Phys. Rev. D 97, 104036 (2018)

[20] F. Douchin and P. Haensel, Astron. Astrophys. 380, 151 (2001)

[21] J. M. Lattimer and F. D. Swesty, Nucl. Phys. A 535, 331 (1991)

[22] H. Shen, H. Toki, K. Oyamatsu, and K. Sumiyoshi, Nucl. Phys. A 637, 435 (1998)
[23] A. Akmal, V. R. Pandharipande, and D. G. Ravenhall, Phys. Rev. C 58, 1804 (1998)

[24] R. B. Wiringa, V. Fiks, and A. Fabrocini, Phys. Rev. C 38, 1010 (1988)

[25] L. Engvik, E. Osnes, M. Hjorth-Jensen, G. Bao, and E. Ostgaard, Astrophys. J. 469, 794 (1996)

[26] H. Müther, M. Prakash, and T. L. Ainsworth, Phys. Lett. B 199, 469 (1987)

[27] H. Müller and B. D. Serot, Nucl. Phys. A 606, 508 (1996)

[28] J. Antoniadis, P. C. C. Freire, et al., Science 340, 1233232 (2013)

[29] T. Zhao and J. M. Lattimer, Phys. Rev. D 98, 063020 (2018)

[30] B. P. Abbott et al. (LIGO Scientific Collaboration and Virgo Collaboration), Phys. Rev. Lett. 116, 241103 (2016)

[31] B. P. Abbott et al. (LIGO Scientific Collaboration and Virgo Collaboration), Phys. Rev. X 6, 041015 (2016)

[32] S. De, D. Finstad, J. M. Lattimer, D. A. Brown, E. Berger, and C. M. Biwer, Phys. Rev. Lett. 121, 259902 (2018)

[33] M. Agathos, J. Meidam, W. Del Pozzo, T. G. F. Li, M. Tompitak, J. Veitch, S. Vitale, and C. Van Den Broeck, Phys. Rev. D 92, 023012 (2015)

[34] LIGO Scientific Collaboration, "LIGO Algorithm Library LALSuite," ree software (GPL) (2018).

[35] L. Barsotti, S. Gras, M. Evans, and P. Fritschel, The updated Advanced LIGO design curve, Tech. Rep. LIGO-T1800044 (The LIGO Scientific Collaboration, 2018).

[36] T. Dietrich, S. Bernuzzi, and W. Tichy, Phys. Rev. D 96, 121501 (2017)

[37] J. Vines, E. E. Flanagan, and T. Hinderer, Phys. Rev. D 83, 084051 (2011)

[38] D. Radice, A. Perego, F. Zappa, and S. Bernuzzi, Astrophys. J. Lett. 852, L29 (2018)

[39] K. Kiuchi, K. Kyutoku, M. Shibata, and K. Taniguchi, Astrophys. J. Lett. 876, L31 (2019) 